\title{
Understanding new-onset refractory status epilepticus from an immunological point of view
}

\author{
Yong-Won Shin \\ Center for Hospital Medicine, Department of Neurosurgery, Seoul National University Hospital, Seoul, Korea
}

\begin{abstract}
New-onset refractory status epilepticus (NORSE) is unexpected onset of refractory status epilepticus in individuals with no preexisting relevant neurologic condition. The etiologies remain largely cryptogenic; treatment is challenging after failure to control seizures despite use of multiple antiepileptic drugs and anesthetic agents. Frequent fever and other infectious prodromes, elevated proinflammatory cytokine/chemokine levels, and limbic or multifocal brain lesions indicate active inflammation in NORSE. Among identified causes, autoimmune encephalitis is the most common and accounts for more than one-third of all known NORSE cases, followed by infection-related etiologies. Although more evidence is needed, anti-cytokine therapies with tocilizumab and anakinra along with other immunotherapeutic agents used in autoimmune encephalitis can aid in alleviating or hindering the inflammatory cascade and controlling seizures.
\end{abstract}

Keywords: New-onset refractory status epilepticus, Etiology, Autoimmune, Inflammation, Cryptogenic

\section{Introduction}

Status epilepticus (SE) is an emergent and severe neurologic condition involving persistent or recurrent seizures. Approximately $30 \%$ to $40 \%$ of patients with SE are refractory (RSE) to first- and second-line treatments, which results in high mobility and mortality [1-3]. New-onset RSE (NORSE) refers to a specific clinical presentation where RSE occurs in patients without active epilepsy or other preexisting relevant neurologic disorders and without clear acute or active structural, toxic, or metabolic causes [4,5]. Management of NORSE is challenging because its underlying etiology is not identifiable readily, and antiepileptic drugs and anesthetic agents often fail to control seizures $[4,6,7]$. Although the etiologies of NORSE remain cryptogenic in most cases, autoimmune and infectious factors are present in at least $30 \%$ to $40 \%$ of cases, and fever or other infectious prodrome is frequent, suggesting an immune-mediated pathogenesis [4,6-8]. This review introduces neuroinflammatory perspectives of NORSE from etiology to therapeutic strategies.

\section{Neuroinflammatory etiology in new-onset refractory status epilepticus}

Several studies have reported that more than half of RSE cases have an acute symptomatic etiology, especially encephalitis or inflammatory etiologies [2,3,9]. NORSE excludes structural, toxic, and metabolic causes by consensus definition [5]; in addition, acute symptomatic etiologies (including infection and inflammation) account for a larger proportion of NORSE than of RSE cases in general.

In several studies, non-paraneoplastic and paraneoplastic autoimmune encephalitis have been the most frequently identified causes of NORSE $[4,7,8,10]$. Autoantibodies to the $N$-methyl-D-aspartate (NMDA) receptor are the most common among identified autoantibodies in autoimmune encephalitis and frequently are identified as a cause of NORSE along with encephalitis associated with antibodies to the voltage-gated potassium channel complex, which are distinguished into anti-leucine-rich

Received: March 6, 2021 Revised: April 11, 2021 Accepted: May 21, 2021

Correspondence: Yong-Won Shin

Center of Hospital Medicine, Department of Neurosurgery, Seoul National University Hospital, 101 Daehak-ro, Jongno-gu, Seoul 03080, Korea

E-mail: shinwn04@gmail.com

ORCID: https://orcid.org/0000-0002-6781-7782

Copyright (C) 2021 by The Korean Encephalitis and Neuroinflammation Society

This is an open access article distributed under the terms of the Creative Commons Attribution Non-Commercial License (http://creativecommons.org/licenses/by-nc/4.0/) which permits unrestricted non-commercial use, distribution, and reproduction in any medium, provided the original work is properly cited. 
glioma-inactivated 1 (LGI1) and anti-contactin associated protein 2 (CASPR2) [4].

In more than half of NORSE cases, the etiology is unclear, and these cases are categorized as cryptogenic $[4,7]$. One reason for the large proportion of cryptogenic cases could be a less extensive work-up. Recently, the etiology in three of 26 NORSE patients was discovered only after brain biopsy and/or autopsy [7]. Autoimmune encephalitis associated with unidentified antibodies could be another cause of cryptogenic NORSE. However, a study by lizuka et al. [6] that compared cryptogenic NORSE with anti-NMDA receptor encephalitis showed distinguishing clinical and immunological features. In that study, patients with cryptogenic NORSE demonstrated less cerebrospinal fluid (CSF) pleocytosis, no oligoclonal bands in the CSF, and a rarely elevated immunoglobulin G (IgG) index. Immunohistochemistry using a rat brain or hippocampal culture also did not show evidence of antineuronal antigens. Clinically, there were no psychobehavioral or memory alterations and infrequent involuntary movements. However, that study also found that most patients with cryptogenic NORSE had prodromal fever and brain magnetic resonance imaging (MRI) abnormalities, and median protein levels in the CSF were higher than those typically seen in anti-NMDA receptor encephalitis, indicating an underlying inflammation-mediated mechanism.

Febrile infection-related epilepsy syndrome (FIRES) is a subcategory of NORSE that presents with a prior febrile infection occurring between 2 weeks and 24 hours prior to onset of RSE [5]. FIRES often is discussed separately from NORSE because of its distinct age distribution (mostly in children) and clear history of febrile illness. However, the clinical manifestations, disease course, and outcomes are similar in FIRES and other types of NORSE, and these two conditions are considered to have a largely shared pathophysiology. A report of an association between FIRES and an ILIRN gene polymorphism supports an inflammation-mediated pathogenesis [11]. That study also suggests that more investigation is needed into genetic factors that affect the immune response in such patients.

SE is associated with several preinflammatory pathways, such as the nuclear factor- $\mathrm{kB}$ (NF-kB) signaling pathway, the mechanistic target of rapamycin (mTOR) signaling pathway, the mitogen-activated protein kinase (MAPK) signaling pathway, and the transforming growth factor- $\beta$ (TGF- $\beta$ ) signaling pathway [12]. Previous studies that have examined the serum and CSF in NORSE and FIRES patients have reported upregulation of multiple cytokines/chemokines [13-15]. TGF- $\beta$ signaling pathways also are involved in blood-brain barrier (BBB) disruption, which contributes to SE and epileptogenesis [16]. More than half of NORSE patients showed MRI abnormalities at the initial or follow-up evaluation and also frequently demonstrated limbic area involvement $[4,6-8,17]$. Leptomeningeal enhancement indicating BBB disruption has been reported in NORSE patients at variable proportions and is associated with both pharmacoresistance and poor functional outcome $[4,8,17]$.

Therefore, autoimmune and other neuroinflammatory etiologies mainly are involved in NORSE pathogenesis, and the initial noninflammatory neurological condition can accompany neuroinflammation under SE and involve epileptogenesis due to BBB disruption and excitotoxic neuronal damage, which all lead to a fulminant inflammatory response in the brain.

\section{Etiologic evaluation of NORSE}

Initial patient evaluation includes brain MRI, electroencephalogram, CSF study, and blood analysis. Brain MRI can identify structural lesions or show evidence of neuroinflammation. CSF pleocytosis and elevated CSF protein levels can aid in identifying neuroinflammation during its initial stages. Autoimmune and infectious causes must be evaluated thoroughly considering the large number of identifiable etiologies. A list of suggested screening investigations in NORSE patients is summarized in Table 1. A cytokine assay of serum and CSF can provide supportive evidence of neuroinflammation and aid in therapeutic decision-making. If cancer is suspected, a computed tomography scan of the chest, abdomen, and pelvis or whole-body positron emission tomography should be conducted. Pelvic MRI is a feasible alternative for diagnosing ovarian teratoma if anti-NMDA receptor encephalitis is suspected. Metabolic and toxicological screening and genetic investigation also can help identify noninflammatory causes of NORSE. If there is an identifiable brain lesion of unknown etiology on MRI, brain biopsy can be indicated.

\section{Immunologic treatment in NORSE}

NORSE occurs in individuals without a previous history of epilepsy or other neurologic conditions and often presents with a prodromal phase followed by a progressive build-up of seizures. Initial MRIs often show no abnormality, but subsequent limbic or extra-limbic lesion development has been reported. Considering its clinical course, NORSE is classified as a rapid progressive encephalitic condition accompanied by uncontrolled seizures. Resistance to antiepileptic drugs is an important characteristic of epilepsy with an autoimmune etiology [18], and active 
Table $1 \mathrm{~A}$ list of suggested screening investigations in NORSE

\begin{tabular}{|c|c|}
\hline \multirow[t]{5}{*}{ Autoimmune } & Antineuronal surface autoantibodies: NMDAR, LGI1, CASPR2, GABA $R, \mathrm{AABA}_{B} \mathrm{R}, \mathrm{AMPAR}$, glycine receptor \\
\hline & Paraneoplastic/intracellular antigens: Hu, Yo, Ri, CRMP5, Ma2, GAD65, and amphiphysin \\
\hline & Other serologies: ANA, ANCA, anti-thyroid antibodies \\
\hline & Oligoclonal band \\
\hline & Might extend to other known autoantibodies associated with CNS disorders \\
\hline \multirow[t]{6}{*}{ Infectious } & CSF PCR for HSV1/2, VZV, EBV, HIV \\
\hline & Bacterial and fungal stains and cultures \\
\hline & Work-up for mycobacterial tuberculosis \\
\hline & VDRL \\
\hline & $\begin{array}{l}\text { Might extend to other neuroinfectious agents. A more extensive list should be considered for immunocompromised patients including } \\
\text { Cryptococcus species, Toxoplasma gondii, Histoplasma capsulatum, JC virus, CMV, HHV6, parvovirus, and WNV (endemic areas) }\end{array}$ \\
\hline & Also consider metagenomic study to identify possible infectious etiologies \\
\hline \multicolumn{2}{|c|}{$\begin{array}{l}\text { NORSE, new-onset refractory status epilepticus; NMDAR, N-methyl-D-aspartate receptor; LGI1, leucine-rich glioma-inactivated 1; CASPR2, contactin associated protein } \\
\text { 2; GABA }{ }_{A} R \text {, gamma-aminobutyric acid A receptor; GABA }{ }_{B} R \text {, GABA B receptor; AMPAR, } \alpha \text {-amino-3-hydroxy-5-methyl-4-isoxazolepropionic acid; CRMP5, collapsin response } \\
\text { mediator protein 5; GAD65, glutamic acid decarboxylase; ANA, antinuclear antibodies; ANCA, antineutrophil cytoplasmic antibodies; CNS, central nervous system; CSF, } \\
\text { cerebrospinal fluid; PCR, polymerase chain reaction; HSV, herpes simplex virus; VZV, varicella-zoster virus; EBV, Epstein-Barr virus; HIV, human immunodeficiency virus; } \\
\text { VDRL, venereal disease research laboratory; CMV, cytomegalovirus; HHV6, human herpesvirus 6; WNV, West-Nile virus. }\end{array}$} \\
\hline
\end{tabular}

inflammation is observed in drug-resistant epilepsy and RSE of diverse causes [19]. Therefore, early initiation of immunotherapy can aid in alleviating or hindering the inflammatory cascade in the brain and reducing irreversible damage. However, evidence is lacking on whether timely intervention for neuroinflammation can alter the disease course and outcomes in NORSE.

Typical initial treatments include broad immune suppression and BBB stabilization with a steroid and neutralizing and competing potential autoantibodies along with normalized immune milieu using intravenous Ig and/or plasma exchanges. Rituximab, which targets CD20-expressing B cells, and lymphocyte-targeting antiproliferative agents (such as cyclophosphamide, mycophenolate mofetil, and azathioprine) are among the available choices in cases with persistent seizures. These strategies are based on immunotherapies that have been successfully administered to autoimmune encephalitis patients [20].

Tocilizumab is a monoclonal antibody that targets the interleukin (IL)-6 receptor. An investigation into the increase in proinflammatory cytokines in adult patients with NORSE showed substantial upregulation of IL-6 along with significant increase in IL-2, IL-4, IL-5, IL-10, and tumor necrosis factor- $\alpha$ (TNF $\alpha$ ) in the CSF [13]. In this prior study, tocilizumab successfully controlled SE in six of seven patients. A study of acute encephalitis patients with refractory repetitive partial seizures revealed upregulation of IL-6, C-X-C motif chemokine (CXCL) 10, and IL-8 in the CSF [14]. Another study that investigated FIRES also showed increase in $\mathrm{T}$ helper cell type 1-associated cytokines/ chemokines (TNF $\alpha$, CXCL9, CXCL10, and CXCL11), IL-6, C-C motif ligand (CCL) 2, CCL19, and CXCL1 [15]. IL-6 is one of the most important proinflammatory mediators in neuroinflammation; previous studies consistently have reported upregulation of IL-6 in NORSE/FIRES patients, supporting the use of tocilizumab. However, further studies evaluating the therapeutic benefit of tocilizumab in NORSE are needed.

Anakinra is an IL-1 receptor antagonist that inhibits the biological actions of IL- $1 \beta$ and is used in a variety of autoinflammatory disorders [21]. Along with IL-6, IL-1 $\beta$ is implicated in neuronal hyperexcitability and seizures. After the first case report of the effectiveness of anakinra in FIRES [22], subsequent publications have supported the therapeutic effect of anakinra in FIRES patients [23-25]. However, another study found that administration of anakinra did not control seizures in a FIRES patient before additional treatment with tocilizumab [26]. In addition, increase of IL- $1 \beta$ has not been consistent in previous reports [15,22,23], although downstream proinflammatory cytokines/chemokines induced by IL-1 $\beta$ often are elevated in these patients. There is a lack of reporting of anakinra treatment in NORSE patients without FIRES.

Tofacitinib is a Janus kinase (JAK) inhibitor and can be used in several autoimmune and inflammatory disorders, including rheumatoid arthritis, psoriatic arthritis, and ulcerative colitis [27]. Tofacitinib has been reported to penetrate the BBB and exert a potentially therapeutic effect in neuroinflammatory conditions [28,29]. Recently, tofacitinib was administered to NORSE patients in a study in which two of the eight NORSE patients showed a good response [30]. Further studies are needed to replicate these results and determine whether administration of this drug could increase the efficacy of treatment. 


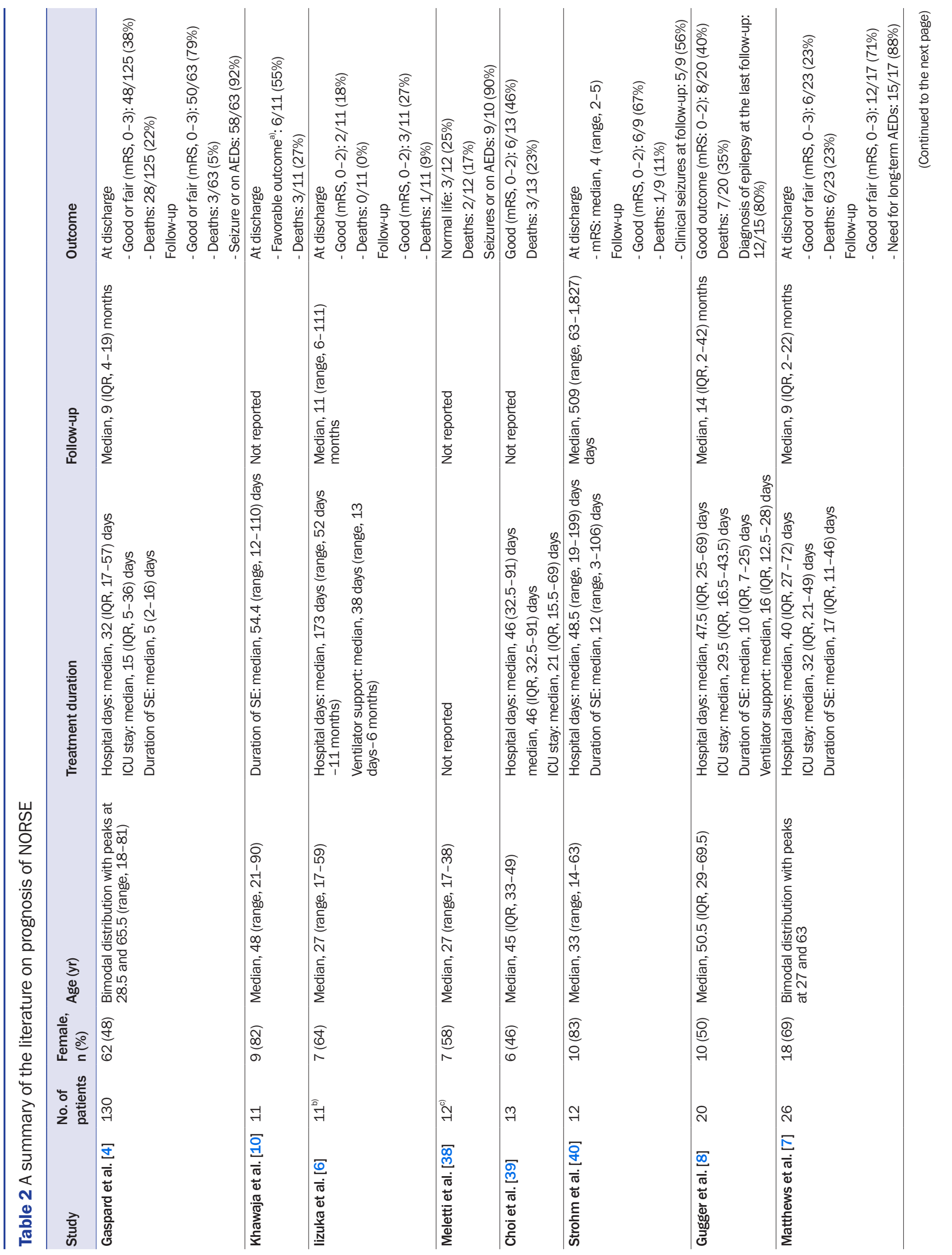




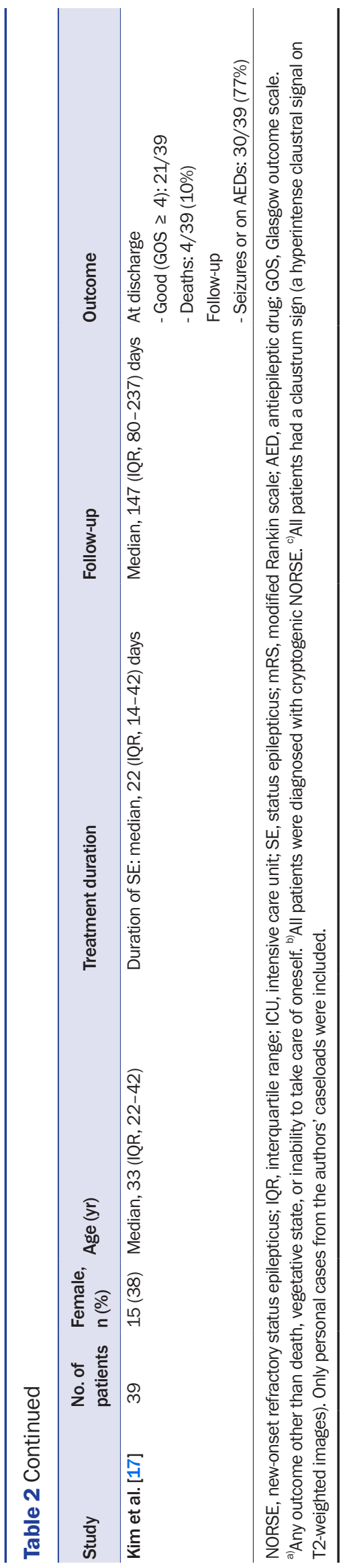

A ketogenic diet is thought to have both anti-inflammatory and neuroprotective effects along with anti-seizure effects [19]. Many reports have shown that a ketogenic diet can aid in seizure control and improve cognitive outcomes in FIRES [31-33]. Investigations into use of a ketogenic diet in NORSE patients have suggested a potential therapeutic benefit $[8,34]$.

Vagus nerve stimulation (VNS) also can modulate the immune response via a cholinergic anti-inflammatory pathway [35]. A case report demonstrated a reduction in seizure activity with VNS in NORSE as well as other RSE patients [36,37].

\section{Outcomes in NORSE patients}

The outcomes in NORSE patients can vary by etiology and treatment, but full recovery is infrequent. Many survivors suffer from long-term cognitive and behavioral sequelae in addition to epilepsy. The reported mortality is substantial, ranging from $5 \%$ to $35 \%$. Among survivors, $56 \%$ to $92 \%$ continue to experience seizures or need to be maintained on antiepileptic drugs $[4,6$ $8,10,17,38-40]$. All prior reports with more than 10 cases of adult NORSE patients are summarized in Table 2.

\section{Conclusion}

NORSE is a devastating condition that is refractory to antiepileptic and anesthetic medications. Poor understanding of the pathophysiologic mechanism combined with unknown and potentially diverse, rare etiologies often complicate identification of treatments optimized by patient, resulting in substantial morbidity and high mortality. However, recent progress in the understanding of autoimmune encephalitis has aided in additional identification of etiologies in NORSE patients and has led to new treatment options, including immunomodulatory therapies. Active investigation into the etiology of NORSE will help further establish a treatment plan. Brain biopsy and genetic investigations could help provide clues to the pathogenesis of this condition in some cases. Further investigation is needed to extend our understanding of the causes of NORSE and to accumulate more evidence for evaluating the efficacies of different immunomodulatory therapies.

\section{Conflicts of Interest}

No potential conflict of interest relevant to this article was reported. 


\section{Acknowledgments}

This work was supported by a National Research Foundation of Korea (NRF) grant funded by the Korean government (MSIT) (No. 2020R1C1C1014982).

\section{References}

1. Mayer SA, Claassen J, Lokin J, Mendelsohn F, Dennis LJ, Fitzsimmons BF. Refractory status epilepticus: frequency, risk factors, and impact on outcome. Arch Neurol 2002;59:205-210.

2. Holtkamp M, Othman J, Buchheim K, Meierkord H. Predictors and prognosis of refractory status epilepticus treated in a neurological intensive care unit. J Neurol Neurosurg Psychiatry 2005;76:534-539.

3. Delaj L, Novy J, Ryvlin P, Marchi NA, Rossetti AO. Refractory and super- refractory status epilepticus in adults: a 9-year cohort study. Acta Neurol Scand 2017;135:92-99.

4. Gaspard N, Foreman BP, Alvarez V, et al. New-onset refractory status epilepticus: Etiology, clinical features, and outcome. Neurology 2015;85:1604-1613.

5. Hirsch LJ, Gaspard N, van Baalen A, et al. Proposed consensus definitions for new-onset refractory status epilepticus (NORSE), febrile infection-related epilepsy syndrome (FIRES), and related conditions. Epilepsia 2018;59:739-744.

6. Iizuka T, Kanazawa N, Kaneko J, et al. Cryptogenic NORSE: Its distinctive clinical features and response to immunotherapy. Neurol Neuroimmunol Neuroinflamm 2017;4:e396.

7. Matthews E, Alkhachroum A, Massad N, et al. New-onset super-refractory status epilepticus: a case series of 26 patients. Neurology 2020;95:e2280-e2285.

8. Gugger JJ, Husari K, Probasco JC, Cervenka MC. New-onset refractory status epilepticus: a retrospective cohort study. Seizure 2020;74:41-48.

9. Vooturi S, Jayalakshmi S, Sahu S, Mohandas S. Prognosis and predictors of outcome of refractory generalized convulsive status epilepticus in adults treated in neurointensive care unit. Clin Neurol Neurosurg 2014;126:7-10.

10. Khawaja AM, DeWolfe JL, Miller DW, Szaflarski JP. New-onset refractory status epilepticus (NORSE)--The potential role for immunotherapy. Epilepsy Behav 2015;47:17-23.

11. Saitoh M, Kobayashi K, Ohmori I, et al. Cytokine-related and sodium channel polymorphism as candidate predisposing factors for childhood encephalopathy FIRES/AERRPS. J Neurol Sci 2016;368:272-276.

12. Wang M, Chen Y. Inflammation: a network in the pathogenesis of status epilepticus. Front Mol Neurosci 2018;11:341.

13. Jun JS, Lee ST, Kim R, Chu K, Lee SK. Tocilizumab treatment for new onset refractory status epilepticus. Ann Neurol 2018;84:940-945.

14. Sakuma H, Tanuma N, Kuki I, Takahashi Y, Shiomi M, Hayashi M. Intrathecal overproduction of proinflammatory cytokines and chemok- ines in febrile infection-related refractory status epilepticus. J Neurol Neurosurg Psychiatry 2015;86:820-822.

15. Kothur K, Bandodkar S, Wienholt L, et al. Etiology is the key determinant of neuroinflammation in epilepsy: elevation of cerebrospinal fluid cytokines and chemokines in febrile infection-related epilepsy syndrome and febrile status epilepticus. Epilepsia 2019;60:1678-1688.

16. Gorter JA, van Vliet EA, Aronica E. Status epilepticus, blood-brain barrier disruption, inflammation, and epileptogenesis. Epilepsy Behav 2015;49:13-16

17. Kim HJ, Lee SA, Kim HW, Kim SJ, Jeon SB, Koo YS. The timelines of MRI findings related to outcomes in adult patients with new-onset refractory status epilepticus. Epilepsia 2020;61:1735-1748.

18. Quek AM, Britton JW, McKeon A, et al. Autoimmune epilepsy: clinical characteristics and response to immunotherapy. Arch Neurol 2012; 69:582-593.

19. Koh S, Dupuis N, Auvin S. Ketogenic diet and Neuroinflammation. Epilepsy Res 2020;167:106454.

20. Shin YW, Lee ST, Park KI, et al. Treatment strategies for autoimmune encephalitis. Ther Adv Neurol Disord 2017;11:1756285617722347.

21. Dinarello CA, Simon A, van der Meer JW. Treating inflammation by blocking interleukin-1 in a broad spectrum of diseases. Nat Rev Drug Discov 2012;11:633-652

22. Kenney-Jung DL, Vezzani A, Kahoud RJ, et al. Febrile infection-related epilepsy syndrome treated with anakinra. Ann Neurol 2016;80:939945.

23. Clarkson BD, LaFrance-Corey RG, Kahoud RJ, Farias-Moeller R, Payne ET, Howe CL. Functional deficiency in endogenous interleukin-1 receptor antagonist in patients with febrile infection-related epilepsy syndrome. Ann Neurol 2019;85:526-537.

24. Dilena R, Mauri E, Aronica E, et al. Therapeutic effect of Anakinra in the relapsing chronic phase of febrile infection-related epilepsy syndrome. Epilepsia Open 2019;4:344-350.

25. Lai YC, Muscal E, Wells E, et al. Anakinra usage in febrile infection related epilepsy syndrome: an international cohort. Ann Clin Trans Neurol 2020;7:2467-2474.

26. Stredny CM, Case S, Sansevere AJ, Son M, Henderson L, Gorman MP. Interleukin-6 blockade with tocilizumab in anakinra-refractory febrile infection-related epilepsy syndrome (FIRES). Child Neurol Open 2020;7:2329048X20979253.

27. Jamilloux Y, El Jammal T, Vuitton L, Gerfaud-Valentin M, Kerever S, Sève P. JAK inhibitors for the treatment of autoimmune and inflammatory diseases. Autoimmun Rev 2019;18:102390.

28. Fukuyama T, Tschernig T, Qi Y, Volmer DA, Bäumer W. Aggression behaviour induced by oral administration of the Janus-kinase inhibitor tofacitinib, but not oclacitinib, under stressful conditions. Eur J Pharmacol 2015;764:278-282.

29. Haile WB, Gavegnano C, Tao S, Jiang Y, Schinazi RF, Tyor WR. The Ja- 
nus kinase inhibitor ruxolitinib reduces HIV replication in human macrophages and ameliorates HIV encephalitis in a murine model. Neurobiol Dis 2016;92(Pt B):137-143.

30. Jang Y, Lee WJ, Lee HS, Chu K, Lee SK, Lee ST. Tofacitinib treatment for refractory autoimmune encephalitis. Epilepsia 2021;62:e53-e59.

31. Nabbout R, Mazzuca M, Hubert P, et al. Efficacy of ketogenic diet in severe refractory status epilepticus initiating fever induced refractory epileptic encephalopathy in school age children (FIRES). Epilepsia 2010;51:2033-2037.

32. Singh RK, Joshi SM, Potter DM, Leber SM, Carlson MD, Shellhaas RA. Cognitive outcomes in febrile infection-related epilepsy syndrome treated with the ketogenic diet. Pediatrics 2014;134:e1431-e1435.

33. Peng P, Peng J, Yin F, et al. Ketogenic diet as a treatment for super-refractory status epilepticus in febrile infection-related epilepsy syndrome. Front Neurol 2019;10:423.

34. Thakur KT, Probasco JC, Hocker SE, et al. Ketogenic diet for adults in super-refractory status epilepticus. Neurology 2014;82:665-670.

35. Bonaz B, Sinniger V, Pellissier S. Anti-inflammatory properties of the vagus nerve: potential therapeutic implications of vagus nerve stimulation. J Physiol 2016;594:5781-5790.

36. Yamazoe T, Okanishi T, Yamamoto A, et al. New-onset refractory status epilepticus treated with vagus nerve stimulation: a case report. Seizure 2017;47:1-4.

37. Kurukumbi M, Leiphart J, Asif A, Wang J. Vagus nerve stimulation (VNS) in super refractory new onset refractory status epilepticus (NORSE). Case Rep Neurol Med 2019;2019:7852017.

38. Meletti S, Giovannini G, d'Orsi G, et al. New-onset refractory status epilepticus with claustrum damage: definition of the clinical and neuroimaging features. Front Neurol 2017;8:111.

39. Choi JY, Kim EJ, Moon SY, Kim TJ, Huh K. Prognostic significance of subsequent extra-temporal involvement in cryptogenic new onset refractory status epilepticus (NORSE) initially diagnosed with limbic encephalitis. Epilepsy Res 2019;158:106215.

40. Strohm T, Steriade C, Wu G, Hantus S, Rae-Grant A, Larvie M. FDGPET and MRI in the evolution of new-onset refractory status epilepticus. AJNR Am J Neuroradiol 2019;40:238-244. 